\begin{tabular}{|c|l|}
\hline Title & $\begin{array}{l}\text { On the outer large scale motions of wall turbulence and their interaction with near-wall structures using large eddy } \\
\text { simulation }\end{array}$ \\
\hline Author(s) & Tsubokura, Makoto \\
\hline Citation & $\begin{array}{l}\text { Computers \& Fluids, 38(1), 37-48 } \\
\text { https://doi.org/10.1016/.complluid.2007.10.018 }\end{array}$ \\
\hline Issue Date & 2009-01 \\
\hline Doc URL & http:/hdl.handle.net/2115/49858 \\
\hline Type & article (author version) \\
\hline File Information & CaF381_37-48.pdf \\
\hline
\end{tabular}

Instructions for use 


\title{
On the outer large-scale motions of wall turbulence and their interaction with near-wall structures using large eddy simulation
}

\author{
Makoto Tsubokura \\ Department of Mechanical Engineering and Intelligent Systems, \\ The University of Electro-Communications, \\ 1-5-1 Chofugaoka, Chofu-shi, Tokyo, 182-8585, Japan
}

\begin{abstract}
Large-scale structures typically observed at or above the logarithmic layer of fully developed turbulent channel flows were numerically studied. The potential validity of large eddy simulation (LES) to the large-scale analysis was focused on, and its applicability was investigated for the first time by carrying out an intensive grid resolution study to determine the minimum grid spacing necessary to properly capture such flows. It was found that rather fine grid spacing sufficient to resolve the near-wall streaky motions represented by $\lambda_{x}^{+} \times \lambda_{z}^{+} \sim 1000 \times 100$ is required to reproduce the typical spectral features of large structures in the outer layer. Subsequently, the Reynolds-number scaling for such structures and their interaction with buffer-layer turbulence were examined. It was observed that the large structures in the outer layer remarkably appear only in the streamwise velocity fluctuation, basically obeying the outer scaling, and their spanwise size is approximately twice as large as the boundary-layer thickness, independent of the Reynolds-number range tested here. It was also found that they penetrate deep into the
\end{abstract}


buffer layer, where small streaky structures obey the inner scaling. These results clearly demonstrate that mixed inner-outer scaling instead of simple inner scaling for the streamwise velocity root mean square in the near-wall region is reasonable.

Key words: Wall turbulence, Large eddy simulation, Channel flow, Large-scale structure, Organized structure

PACS:

\section{Introduction}

It has been widely acknowledged that in wall turbulence, large-scale structures of a size at least comparable to the boundary layer thickness exist. In contrast to the small-scale structures near the wall, less attention has been paid to the large structures until recently. Del Álamo and Jiménez (2001) argued that one of the reasons for this relative neglect originates from Townsend's first prediction of the large scales under the attached eddy hypothesis, in which he described them as inactive, in the sense that they do not contain Reynolds stress. Later, however, Jiménez (1998) showed from various existing experimental and numerical data that they are rather active, containing substantial Reynolds stress as well as turbulence energy, which has been verified by direct numerical simulation (DNS) of plane channels (Del Álamo and Jiménez, 2001). In this context, interaction between these large scales in the outer layer and the small scales near the wall appears to be an important issue in understanding the Reynolds-number effect on wall turbulence. In fact, the Reynolds-number dependence of streamwise velocity fluctuations near the wall could be explained as a contribution of the outer motions to the inner structures, and evidence of this idea has been shown, for example, by de Graaff and Eaton (2000), who successfully demonstrated that the profile of the 
streamwise velocity root mean square (rms) collapses well in inner-outer mixed scaling. Furthermore, Hunt and Morrison (2000) discussed the top-down structures of wall turbulence, in which large-scale eddies in the middle layer impinge at the wall, leading to the generation of small-scale turbulence and some upscale transfer of energy at very high Reynolds numbers, such as those observed in the atmospheric boundary layer.

The existence of large scales has been recognized in experimental results showing an abnormal extent of temporal autocorrelation appearing only in the streamwise velocity component, or of the low wavenumber peak of corresponding premultiplied one-dimensional power spectra. In addition, Kim and Adrian (1999) pointed out the presence of very large-scale motions, which are much larger than those comparable to the boundary layer thickness, based on the power spectra of the streamwise velocity measured in fully developed turbulent pipe flow. From these experimental results, we can suppose that the large-scale structures are streamwise-elongated anisotropic motions similar to the near-wall streaks, but of a size comparable to or even much larger than the boundary layer thickness.

In spite of such importance and recent attention, only limited studies have so far been carried out on large-scale structures. The practical difficulty lies in the fact that a large experimental set-up or a computational domain at sufficiently high Reynolds-number conditions is necessary to properly capture their entire motions. This requirement has been the stumbling block to both experiments and DNS for large-scale studies. Furthermore, the one-point measurements most frequently used in experimental studies have fundamental difficulty in describing the spatial characteristics of large-scale structures. The validity of a spectrum converted from frequency to wavenumber using Taylor's hypothesis for use in large-scale analysis should also be carefully considered. Alternatively, large eddy simulation (LES) 
might be a promising method, in which large and coherent flow structures are directly solved by three-dimensional time-marching numerical simulation, while rather isotropic and universal eddies are only modeled.

The first objective of this study was to investigate the applicability of LES to the study of such large-scale structures in wall turbulence. Section 2 provides the details of our numerical methods, including analysis models, governing equations, discretization, and turbulence modeling, which is validated in detail in Section 3, in which grid resolution studies are performed to determine the minimum resolution required for the reproduction of large scales in fully developed incompressible turbulent plane channels. Section 3 mainly considers the numerical issues, but also sheds light on the physical question as to whether or not the outer large motions are independent of the organized small motions in the inner layer. In fact, it is demonstrated in Section 3 that excessively fine grid resolution compared with the spatial scales of the large structures is required. This result unexpectedly reveals that the small scales near the wall play an important role in the process of producing the large scales in the outer layer, at least within the moderate Reynolds-number range tested here. Section 4 contributes to the second objective of this study: to investigate the Reynolds-number similarity of the large scales, which will be helpful in estimating the self-similarity of velocity fluctuations. Finally, in Section 5 our results are summarized and some conclusions are drawn. 


\section{Numerical methods}

\subsection{Analysis models}

The analysis models adopted in the present work consist of simple flows between two plane surfaces. An open channel flow between solid and free surfaces is considered in Section 4, while a plane channel flow between two solid walls is used in Section 3, where intensive numerical tests are conducted by comparing our LES results with existing careful DNS data (Del Álamo and Jiménez, 2003). The open channels are supposed to more accurately represent typical wall turbulence than the solid plane channels, in the sense that the former avoids the possible effect of the opposite solid wall on the outer motions. The effect becomes more apparent at lower Reynolds numbers and may cause a particular feature appearing only in limited low-Reynolds-number flows (Wei and Willmarth, 1989).

Hereafter, streamwise, normal-wall, and spanwise directions are given as $x, y$, and $z$, respectively. The flow fields are periodic for the streamwise and spanwise directions, and are driven by the uniform pressure gradient force acting in the streamwise direction to obtain a fully developed turbulent state. The no-slip velocity condition is imposed on the solid wall, in which all velocity components are zero on the surface, while the free-slip condition is imposed on the free surface, in which the velocity component normal to the boundary vanishes, along with the zero normal derivative of the tangential velocity. Thus, the grid spacings in the horizontal directions are uniform, while non-uniform grids with a hyperbolic tangent-type stretching function are used in the normal-wall direction.

The important point to note regarding the normal-wall grid resolution is that at 
least five grid points are allocated through the viscous sublayer to capture the rapid growth of the mean velocity in the vicinity of the wall. This means that no artificial boundary conditions, such as those based on some physical scaling laws usually used to save computer memory consumed for the near-wall grids, were adopted in the present work and any asymptotic behavior of the reproduced organized eddies (typically attached eddies) near the wall is supposed to be well captured.

Domain size and grid number are important parameters in the study of large scales. Because we have adopted periodic conditions for both the streamwise and spanwise directions, the largest scales captured by LES are restricted to half of the domain size, which ideally should be greater than the large scales appearing in the real wall turbulence. The domain sizes and grid numbers used in Section 4 are shown in Table 1. It is noteworthy that unexpectedly fine grids of $h_{x}^{+} \sim 30$ and $h_{z}^{+} \sim 20$ are adopted in the present work. The determination of the grid resolution for the large scales is mentioned in Section 3.

\subsection{Governing equations}

The filtered continuity and momentum equations are given by:

$$
\begin{gathered}
\frac{\partial \bar{u}_{i}}{\partial x_{i}}=0 \\
\frac{\partial \bar{u}_{i}}{\partial t}+\frac{\partial \bar{u}_{i} \bar{u}_{j}}{\partial x_{j}}=-\frac{\partial \bar{p}}{\partial x_{i}}+\frac{1}{R e_{\tau}} \frac{\partial^{2} \bar{u}_{i}}{\partial x_{j} \partial x_{j}}-\frac{\partial}{\partial x_{j}} \tau_{i j}+\delta_{i 1},
\end{gathered}
$$

where an overbar denotes the grid filtering operation, and indices $i=1,2$, and 3 represent the directions for $x, y$, and $z$, respectively. Here, $\bar{u}_{i}$ is the grid-scale (GS) velocity and $\bar{p}$ is the GS pressure divided by constant density. The second equation includes an important numerical parameter: The friction Reynolds number 
$R e_{\tau}=u_{\tau} \delta / \nu$, in which $u_{\tau}, v$, and $\delta$ are the friction velocity on the lower wall, the kinetic viscosity, and channel height, respectively. These non-dimensional equations were obtained by adopting the reference length $\delta$, and velocity $u_{\tau}$, to make the external force appearing in the last terms of Eq. (2) a unit value of 1. Hereafter, values normalized by a velocity scale $u_{\tau}$ and a length scale $v / u_{\tau}$ are denoted by superscript + , which is called inner scaling.

The subgrid-scale (SGS) stress in Eq. (2) is expressed as:

$$
\tau_{i j}=\overline{u_{i} u_{j}}-\bar{u}_{i} \bar{u}_{j}
$$

This term is unknown and must be modeled.

\subsection{Discretization}

Governing equations are discretized on the staggered grid system based on the fully conservative finite difference (FD) scheme (Morinishi et al., 1998). The accuracy is fourth order in space, except for the SGS terms, which is determined by a compromise between higher-order requirements for SGS stress not dominated by numerical errors and lower orders for the non-linear term not contaminated by the aliasing error (e.g., Ghosal, 1996). The third-order Runge-Kutta method is primarily adopted for the time marching, and only the second derivative for the normal-wall direction included in the viscous term is treated semi-implicitly using the Crank-Nicolson method for tolerance of the time increment in the numerical simulation. The fractional step method (Dukowicz and Dvinsky, 1992) is used for the velocity-pressure coupling, and the corresponding pressure Poisson equation is solved by the discrete fast Fourier transform (FFT) method for the periodic directions, while the septa-diagonal method is adopted for the normal-wall 
direction. For details of the discretization method, refer to Morinishi et al. (1998).

\subsection{Subgrid-scale modeling}

An isotropic eddy viscosity model developed previously (Tsubokura, 2001) for a dynamic procedure (Germano et al., 1991) using the FD method is adopted in this study, in which the SGS stress and the corresponding subtest-scale (STS) stress, $T_{i j}=\widetilde{\overline{u_{i} u_{j}}}-\widetilde{\bar{u}}_{i} \widetilde{\bar{u}}_{j}$, are modeled as follows:

$$
\begin{aligned}
\tau_{i j}-\frac{1}{3} \delta_{i j} \tau_{k k} & =-2 C \frac{k}{3|\bar{S}|} \bar{S}_{i j}, \\
T_{i j}-\frac{1}{3} \delta_{i j} T_{k k} & =-2 C \frac{K}{3|\tilde{\bar{S}}|} \tilde{\bar{S}}_{i j},
\end{aligned}
$$

where $S_{i j}$ is the strain rate tensor, $S$ is its magnitude given as $S=\left(2 S_{i j} S_{i j}\right)^{1 / 2}$, and an over-tilde denotes the test filtering operation. The $k$ and $K$ in Eqs. (4) and (5) are the SGS and STS turbulence energy, and are modeled by a scale similarity concept (Bardina et al., 1983) as:

$$
\begin{aligned}
& k=\overline{\overline{u_{k}} \overline{u_{k}}}-\overline{\overline{u_{k}}} \overline{\overline{u_{k}}}, \\
& K=\widehat{\overline{\overline{u_{k}}}}, \tilde{\overline{u_{k}}} \tilde{\overline{\overline{u_{k}}}},
\end{aligned}
$$

which have been obtained by considering the consistency of the numerical error in a dynamic procedure. The model coefficient $C$ in Eqs. (4) and (5) is identical and is determined by the dynamic procedure with the least-square method (Lilly, 1992). To avoid any instability induced by possible negative eddy viscosity through negative $C$, an averaging technique proposed in the original procedure is adopted in the homogeneous directions.

A filtering operation such as grid and test filtering must be conducted explicitly to obtain the SGS eddy viscosity. The one-dimensional grid filtering operation 
discretized by the second-order FD method is given as:

$$
\bar{u}\left(X_{I}\right)=u\left(X_{I}\right)+\frac{\bar{\Delta}^{2}}{24} \frac{u\left(X_{I-1}\right)-2 u\left(X_{I}\right)+u\left(X_{I+1}\right)}{h^{2}}
$$

where $X_{I}$ indicates the location of the discretized velocity on the numerical grid and $I$ is its index, $\bar{\Delta}$ is the grid-filter width, and $h$ is the grid width, given as $X_{I}-X_{I-1}$. The discretized test filtering operation is obtained by replacing $\bar{\Delta}$ with $\tilde{\Delta}$. In this study, this explicit spatial filtering is conducted only in the homogeneous directions. Here, we should bear in mind that the ratio of the filter to the grid width, given as $\bar{\Delta} / h$, is the parameter to be determined a priori. For the explicit grid and test filtering operations, we have adopted $(\bar{\Delta} / h)^{2}=4 / 3$ and $(\tilde{\Delta} / h)^{2}=4$, except for the coarsest case of (I) in Table 2, in which a slightly smaller value of $(\bar{\Delta} / h)^{2}=1$ is adopted. The grid filter parameter for each case is optimized so as to agree closely with the DNS results or the empirical law at the logarithmic layer of the mean velocity profile, as indicated in Figs. 1a and 4a. However, a previous work (Tsubokura, 2001) showed that this optimization is not compulsory, and fairly close agreement with the log-law can be achieved by using the value $(\bar{\Delta} / h)^{2}=1 \sim 2$. The most remarkable feature of the SGS model adopted here is its insensitivity to the test filter parameter $\tilde{\Delta} / h$. For details of the derivation of the models and their excellent performance on wall turbulence, refer to Yoshizawa et al. (1996), Tsubokura et al. (2001), and Tsubokura (2001).

\section{Determination of grid resolution}

In contrast to DNS, which requires resolution down to the Kolmogorov length scale to capture the finest turbulence motion, in LES we can determine the minimum scale of the directly resolved turbulence by choosing proper grid 
resolution. In other words, in LES we are required to confirm in detail as to whether the numerical methods adopted properly resolve the target fluid motion. Thus, grid resolution studies in LES are crucial for the numerical issue.

There is another significant reason for performing grid resolution tests in the present work. Considering the fact that the size of the large scales in the outer layer is at least comparable to the boundary layer thickness, and assuming that their outer motions are detached from the wall and self-organized, it is expected that relatively coarse grids will be sufficient to resolve their motions. On the other hand, if they originate from the small organized structures in the vicinity of the wall, such as proposed in the physical model of Adrian et al. (2000), the grids required for large scales will be unexpectedly fine enough to reproduce the near-wall dominant motions. Accordingly, in this section, we try to determine the appropriate grid resolution for the large-scale structures in LES from both physical and numerical points of view.

A moderate friction Reynolds number of 590 is adopted, at which reliable DNS data are provided by Del Álamo and Jiménez (2003) $\left(R e_{\tau}=550\right)$. This DNS is known as the first simulation using a domain size large enough to study the large scales. It was acknowledged in their DNS that the large scales in the outer layer are described as streamwise elongated anisotropic structures with substantial energy only in the streamwise velocity component. In this respect, we focus on the ability of LES with regard to how properly it can reproduce the premultiplied power spectra of the streamwise velocity fluctuations at various grid resolutions, indicated as (I)-(V) in Table 2. Special attention is paid to a low-wavenumber feature of the spectra in the outer layer, where, remarkably, the large scales appear. In all cases, the adopted domain size is the same as that of the DNS, and the only difference between each LES is the grid resolution for the streamwise and 
spanwise directions. No-slip velocity condition is imposed on both walls, and $\delta$ indicates the channel-half width in this section. It should be mentioned that the wall-normal grid spacing of $h_{y}^{+}=1.6 \sim 44.9$, with a hyperbolic-tangent type stretching function, is coarser than those used in DNS. We determined the resolution so that the obtained mean velocity, turbulent intensity, and one-dimensional spectra shown in Figs. $1 \sim 3$ were relatively independent on that at the specific horizontal grid resolution adopted. In other words, the finer horizontal grid spacings than those shown in Table 2 may increase the wall-normal resolution required.

Figure 1 shows the GS turbulence statistics obtained by LES, along with a comparison with those of DNS. The mean velocity profiles show excellent agreement with the results of DNS, even at the coarsest grid spacing of (I), while GS turbulence intensity shows a slight dependence on grid resolution, especially in the buffer layer $\left(10<y^{+}<20\right)$. Overestimation of the peak of the streamwise rms worsens for the coarser grids, which contradicts the theoretical expectation that the ratio of GS to total turbulence intensity decreases for coarser grids.

This rather poor estimation of LES using coarser grids is widely known as one of the drawbacks of isotropic eddy viscosity models at coarser grid resolutions. These results clearly suggest that the coarser grid of (I) only partly resolves the near-wall dominant anisotropic motions, even if its estimation of mean velocity is satisfactory. On the other hand, there is less dependence on grid resolution in the outer layer $\left(y^{+}>100\right)$ and good correlation with the DNS result can be observed. However, this close agreement with DNS does not necessarily mean that the large scales in the outer region are well reproduced, regardless of the grid resolutions tested here. 
The one-dimensional premultiplied power spectra of the streamwise velocity component are shown in Figs. 2 and 3. Before focusing on the outer-layer spectra, where large scales are remarkably observed, let us first discuss the near-wall spectra at $y^{+} \sim 15$, where the well-known buffer-layer streaks appear and the maximum rms of the streamwise velocity tends to be located. The premultiplied spectra obtained by DNS peak at $k_{x} \delta=4.25$ and $k_{z} \delta=27.5$, as observed in Fig. 2a,b. These values are equivalent to $\lambda_{x}^{+} \sim 810$ and $\lambda_{z}^{+} \sim 130$ in the inner scaling, which represents the streamwise and spanwise scales of the buffer-layer streaks, respectively, (e.g., Kim et al., 1987). Unfortunately, the actual LES of coarser grids such as (I) and (II) fails to reproduce the spectral feature of DNS in the resolved range and cut-off SGS energy seems to pile up on the resolved scale. One of the reasons for the appearance of such false spectra at coarse grid resolutions, which are unexpected from an accurate estimation of mean velocity and acceptable turbulence intensity, could be that they are a result of the relatively low accuracy of the estimated SGS stress by the isotropic eddy viscosity, and fine grid spacing sufficient to directly capture the near-wall small-scale motions [namely at least $\Delta_{x}^{+} \sim 30$ and $\Delta_{z}^{+} \sim 20$ used in $\left.(\mathrm{V})\right]$ is necessary to reproduce the near-wall spectral feature. Considering the characteristic scales of the near-wall structures represented as a peak in the spectra, $\lambda_{x}^{+} \sim 810$ and $\lambda_{z}^{+} \sim 130$, the required grid spacing is indeed reasonable.

We now look at the outer-layer spectra $(y / \delta \sim 0.5)$ relating to reproduction of the large scales. As shown in Fig. 3, the DNS spectra peak at $k_{x} \delta \sim 2.3$ and $k_{z} \delta \sim 3.5,4.5$, which amount to $\lambda_{x} \sim 2.8 \delta$ and $\lambda_{z} \sim 1.8 \delta, 1.4 \delta$ in outer scaling. These low-wavenumber energetic modes represent the characteristic sizes of the large scales. Contrary to the optimistic expectation that rather coarser grids such as (I) and (II), which failed to capture the small scales near the wall, might be 
sufficient to reproduce the large scales in this region, they again fail to reproduce the GS spectra and overestimate the peak wavenumber. That is, (I) and (II) underestimate the characteristic size of the large structures observed in DNS. Reproduction of the steep peak in DNS data in the spanwise spectrum is also unsatisfactory and only the moderate peak can be identified at wavenumbers higher than the DNS value. As we see in the next section (see Figs. 11 and 12), both the streamwise and spanwise scales represented by the peak of the premultiplied spectra increase as we move away from the wall. Figs. 2 a and $3 \mathrm{a}$ show that coarse LES of (I) and (II) cannot even qualitatively estimate this trend, and the spectral peak location at $y^{+} \sim 15$ shifts to a higher wavenumber at $y / \delta \sim 0.5$. This poor performance of the coarse LES was improved by increasing the grid resolution, and (IV) and (V) are both qualitatively and quantitatively accurate enough to reproduce the DNS spectra.

According to the results in the context of reproduction of the peak spectra shown above, we can say that at least grid resolution corresponding to (V) is necessary for analysis of the large scales in LES. The requirement for surprisingly fine grid resolution compared with the characteristic sizes of the large scales reflects the fact that the large scales originate from the near-wall motions related to sublayer streaks. Practically speaking, this grid resolution is relatively fine considering engineering or geophysical applications of LES. However, the total grid number for LES is still approximately one-fiftieth of that used in the referenced spectral DNS, and the advantage of LES for studying the large scales is still maintained.

Finally we would like to note that the comparison between LES and DNS would be more proper if DNS data were filtered onto the specific LES grids. However, the shape of the low-pass filter function cannot be estimated theoretically, owing to the contribution of numerical errors included in the finite difference scheme and 
the SGS model itself (Tsubokura, 2006). To avoid this ambiguity, we compared LES results directly with those of DNS. This treatment does not affect the result mentioned above, because the explicit low pass filtering does not change the spectral peak wavenumber.

\section{Reynolds-number effects}

In accordance with the minimum grid resolution determined in Section 3, we conducted open-channel LES at three Reynolds numbers ranging from low to moderate cases to investigate the Reynolds-number scaling of the large-scale motions. The friction Reynolds number $R e_{\tau}$ as an input parameter and the corresponding bulk Reynolds number $\operatorname{Re}_{C}=U_{C} \delta / \nu$ based on velocity on the slip wall are summarized in Table 3.

\subsection{Turbulence statistics}

Before turning to a closer examination of the large scales and their corresponding energy spectra, a few remarks should be made about basic turbulence statistics, such as the mean velocity and the turbulent intensity, with focus on their Reynolds number scaling. As the most notable feature of wall turbulence, there is general agreement that the streamwise mean velocity shows a logarithmic profile around the overlap region between the inner and outer layers, which collapse in inner scaling. The streamwise mean velocity profiles at three different $R e_{\tau}$ of 395,590 , and 1180 obtained by our LES are illustrated in Fig. 4a. They all show logarithmic profiles and agree very closely with the laws of the wall: $U^{+}=y^{+}$below $y^{+} \sim 5$ and $U^{+}=2.5 \ln y^{+}+5.0$ above $y^{+} \sim 40$. 
Unfortunately, in contrast to the successful mean-velocity scaling, there is no general agreement on the scaling of the turbulence intensity in the near-wall region. This is particularly difficult in the streamwise velocity fluctuations, for which it is widely accepted that the simple classical inner scaling is not effective. A relatively successful scaling method was proposed by de Graaff and Eaton (2000), with mixed scaling of the inner and outer layers, and the effect of the inactive motion represented by the outer unit $\left(u_{\tau}\right.$ as a velocity scale and $\delta$ as a length scale) on the inner-layer turbulence was considered.

Figure $4 \mathrm{~b}$ shows the GS velocity rms of all three components obtained by LES. For reference, experimental results at similar Reynolds number by Wei and Willmarth (1989) (solid plane channels) and by de Graaff and Eaton (2000) (flat-plate boundary layer) are presented by symbols. Fairly close agreement is observed between LES at $R e_{\tau}=1180$ and experimental data at $R e_{\tau}=993$, which indicates the validity of our LES.

\subsection{Spectral properties of the velocity fluctuations}

Figure 5 indicates the 1-D streamwise and spanwise premultiplied power spectra of the streamwise velocity fluctuations (hereafter called ' $u$-spectra') near the wall $\left(y^{+} \sim 20\right)$ against the normalized wavelength in inner scaling. It should be recalled here that the power spectrum multiplied by the wave number $k \phi_{u u}$ in the logarithmic plot indicates that the area under the profile is proportional to the power or energy included in the corresponding wavenumber range, and thus the total area over the entire wavenumber (or wavelength) is equivalent to the turbulence energy of the corresponding velocity component. The peaks of the streamwise and spanwise spectra are located at $\lambda_{x}^{+} \sim 1200,1000,950$ and 
$\lambda_{z}^{+} \sim 120,130,120$ at $R e_{\tau}=395,590,1180$, respectively, which represent the characteristic sizes of the low-speed streaks observed at this height. Roughly speaking, their sizes at different Reynolds numbers are nearly constant in inner scaling, even though the streamwise size is slightly large at $R e_{\tau}=395$.

The main key to explain the near-wall Reynolds-number dependence of the streamwise velocity fluctuations appears in the longer wavelength region of the $u$-spectra, which is typically recognized in the spanwise spectra in Fig. $5 \mathrm{~b}$ at $\lambda_{z}^{+}$ above 1000 . We can observe that more energy piles up at higher Reynolds numbers, and steep peaks appear at $\lambda_{z}^{+} \sim 1000$ and 2000 at $R e_{\tau}=590$ and 1180 , respectively. It is obvious that these low-wavelength peaks comparable to the channel height and their related energy rise increase the streamwise turbulent intensity at higher Reynolds-number flow, and thus contribute to the Reynolds-number dependence of the streamwise peak intensity. It is also noteworthy that the spanwise peak wavelengths at $y^{+} \sim 20$ are equivalent to $\lambda_{z} \sim 1.8 \delta$ in outer scaling, and we can observe an intense peak in the outer layer $(y / \delta \sim 0.5)$ at the corresponding wavelength independent of the Reynolds number, as shown in Fig. 6b. Considering the fact that this spanwise peak in the outer layer represents the spanwise size of the large-scale structures existing there, we can explain the peak wavelengths of $\lambda_{z}^{+} \sim 1000$ and 2000 in the near-wall region $\left(y^{+} \sim 20\right)$ as the influence of the outer-layer structures on the inner-layer turbulence. These results clearly suggest that adopting mixed scaling of the inner and outer units for the streamwise turbulence intensity near the wall is reasonable. Del Álamo and Jiménez (2003) recently investigated the premultiplied 2-D spectral autocorrelation function of the streamwise velocity between $y^{+}=15$ and $y / \delta=0.5$ from DNS data at $R e_{\tau}=180$ and 550, and indicated that the outer-layer structures can be decomposed into two types of modes, one of which is located 
around $\lambda_{x}>5 \delta$ and $\lambda_{z} \sim 2 \delta$, which penetrates deep into the buffer region. Our results shown here support their suggestion, and we can state from Fig. 5b that at the higher Reynolds number of 1180, the existence of large scales in the outer layer and their penetration into the inner layer become more obvious and enhanced, which appears as the remarkable energy rise at $R e_{\tau}=1180$ in the long-wavelength region.

It should be noted here that the normal-wall and spanwise velocity components do not show such a large-scale sign in their premultiplied spectra near the wall (data not shown), and in this context we can say that the large-scale effect of the outer layer on the inner layer is a particular feature of the streamwise velocity, which also confirms the experimental observation of the Reynolds-number dependence of the peak of the turbulence intensity appearing particularly in the streamwise velocity.

\subsection{Visualization of the velocity field}

To better understand the large-scale structures of wall turbulence found in the $u$-spectra and suggesting deep motion in the normal-wall direction, instantaneous velocity fields obtained by the LES were visualized. Figure 7 shows gray-scale coded contours of instantaneous velocity fluctuations $u^{\prime} / u_{\tau}, v^{\prime} / u_{\tau}, w^{\prime} / u_{\tau}$ ranging from -6 to 6 on an $x-z$ plane at $y / \delta \sim 0.5$ at $R e_{\tau}=1180$. The horizontal box in the figure covers the whole numerical region, the streamwise span of which is almost 40 -fold $(12 \pi \delta)$ as large as the channel height. We can observe in Fig. 7a coherent streaky structures similar to the low-speed streaks in the near-wall region. However, the spanwise spacing is equivalent in order to the channel height and the streamwise structure spans almost half of the domain, while such large 
organized structures are not obviously evident in the other two velocity components. It is interesting that their structures apparently resemble the well-known large structures in the plane turbulent Couette flow, such as observed by Komminaho et al. (1996).

Instantaneous streamwise velocity fields at two different cross-sections $[y-z$ plane, illustrated in Fig. 7a as $(A)$ and $(B)]$ are visualized in Fig. 8. The figure shows that approximately three or four large lower-velocity zones align in the spanwise direction. Compared with the low-speed streaks evident as very small black dots in the vicinity of the wall, their sizes are remarkably large. It seems that their spanwise interval is approximately twice the channel height, which is identified as a peak in the 1-D premultiplied power spectra shown in Fig. 6b. Another important feature is that the flow field is dominated by only the two typical separate characteristics, the large-scale structures comparable to the boundary-layer thickness and the small scales in the vicinity of the wall, while the hierarchical structures between these two isolated scales are ambiguous. It is also evident that the large scales in the outer layer penetrate deep into the near-wall region, which probably affect the characteristics of the near-wall turbulence. Similar large motions of uniform lower momentum have also been found by Meinhart and Adrian (1995) in a zero-pressure-gradient turbulent boundary layer. They stated that this was induced by packets of hairpin vortices aligning in the streamwise direction (Adrian et al., 2000).

To study the effect of large scales on the buffer-layer structures and the Reynolds-number dependence, the distribution of instantaneous streamwise velocity fluctuations on the simultaneous horizontal plane at $y^{+} \sim 20$ and $y / \delta \sim 0.5$ at three different Reynolds numbers is shown in Figs. 9 and 10, respectively. Because both figures are illustrated for outer scaling, the streak 
structures indicated in Fig. 9 appear to be finer at higher Reynolds numbers. The most interesting feature is the large darker spots (a typical example is indicated as a dotted oval for reference in Fig. 9c), which are closely correlated with the large-scale structures in the outer layer shown in Fig. 10c. As a result of the distinct difference in scale between the small and large structures at higher Reynolds numbers, this feature is most apparent in Fig. 9c, which also clearly suggests that the large scales in the outer layer are one of the main reasons for the dependence of the near-wall turbulence on the Reynolds number.

Contrary to the near-wall structures, the outer-layer structures generally do not show a definite dependence on the Reynolds number, and we cannot identify a notable difference in Fig. 10 among three different Reynolds numbers.

\subsection{Reynolds-number scaling of the peak wavelength}

The most energetic streamwise wavelengths at which the peak of the premultiplied $u$-spectra are located are plotted in Fig. 11 against distance from the wall. Here we do not count the mode at the longest and second-longest resolvable wavelengths, such as shown in Fig. 6a, and only the first distinct maximum identified in the shorter wavelengths is considered to be the peak of the premultiplied spectra.

These streamwise energetic peak wavelengths collapse relatively well by inner scaling near the wall, as observed in Fig. 11a, decaying asymptotically to $\lambda_{x}^{+} \sim 1000$. They are constant in the buffer layer, then increase rapidly from $y^{+}=100$ to 200 until they reach $\lambda_{x}$ of approximately $3-4 \delta$, but their collapse in the outer layer by outer units is only moderate, as indicated in Fig. 11 b.

The spanwise peak wavelengths show better collapse in both inner and outer 
layers than the streamwise ones, as indicated in Fig. 12. In the near-wall region, they decay asymptotically to $\lambda_{z}^{+} \sim 100$. It is acknowledged that this wavelength is the space between each sublayer streak, suggesting that the characteristic scale of streaks resulting from near-wall coherent eddy structures is a universal phenomenon, even though the long-wavelength motions of the near-wall structures are Reynolds-number-dependent, as observed in Fig. 5b. They grow in the buffer layer, with good collapse in inner scaling, and reach constant values of $\lambda_{z} \sim 1.8 \delta$ around $y^{+} \sim 100$, which then collapse very well in outer scaling.

\section{Conclusion}

We performed LES of turbulent channel flows using a large numerical box to investigate large outer structures. An unexpectedly fine grid spacing of $h_{x}^{+} \sim 30$ and $h_{z}^{+} \sim 20$ in the streamwise and spanwise directions was required to properly reproduce the 1-D premultiplied power spectra and their peak at the lower wavenumber in the outer layer, where large scales remarkably appear. This result suggests a strong relation between the near-wall small scales and the large scales in the outer layer, and supports the physical models of Adrian et al. (2000), in which large-scale motions are induced by subsequent motion down to the near-wall turbulence. Del Álamo and Jiménez (2006) also mentioned the existence of vortex clusters which links the small scales near the wall to the larger ones far from it, using their DNS data of plane channels at the friction Reynolds number up to 1900 . The clusters are self-similar reaching from the logarithmic layer to the near wall region as attached eddies with their length scale proportional to the wall distance of their centers. The result also validates the strong inner-outer interaction suggested in this study. 
The streamwise and spanwise peak wavelength of the premultiplied spectra collapsed very well near the wall in inner scaling, while at or above the logarithmic layer, their peak wavelengths reached maxima of $\lambda_{x} \sim 3 \delta$ and $\lambda_{z} \sim 2 \delta$, independent of the Reynolds numbers tested here.

The reasonable description of near-wall turbulence suggested by our results is that near-wall turbulence consists of low-speed streaks characterized as $\lambda_{x}^{+} \times \lambda_{z}^{+} \sim 1000 \times 100$, which is fundamentally independent of the Reynolds number, but is perturbed by large outer-scale turbulence motions at higher Reynolds numbers. These large outer-scale motions appear as large elongated spots of lower momentum zones, which is one of the important features of high-Reynolds-number wall turbulence.

\section{Acknowledgements}

This work was supported in part by a Grant-in-Aid for Scientific Research from the Ministry of Education, Science, Sports and Culture of Japan (No. 14750110). The DNS data provided by J.C. Del Álamo and J. Jiménez are gratefully acknowledged. We are also indebted to Dr. Y. Morinishi for providing part of the simulation code used here. 


\section{References}

Adrian, RJ, Meinhart, CD, Tomkins, CD. Vortex organization in the outer region of the turbulent boundary layer. J Fluid Mech 2000; 422: 1-54.

Bardina, J, Ferziger, JH, Reynolds, WC. Improved turbulence models based on large eddy simulation of homogeneous, incompressible turbulent flows. Stanford University Report TF-19 1983.

De Graaff, DB, Eaton, JK. Reynolds-number scaling of the flat-plate turbulent boundary layer. J Fluid Mech 2000; 422: 319-46.

Del Álamo, JC, Jiménez, J. Direct numerical simulation of the very large anisotropic scales in a turbulent channel. Center for Turbulence Research Annual Research Briefs, Stanford University 2001; 329-41.

Del Álamo, JC, Jiménez, J. Spectra of the very large anisotropic scales in turbulent channels. Phys Fluids 2003; 15: L41-4.

Del Álamo, JC, Jiménez, J, Zandonade, P, Moser, R. Self-similar vortex clusters in the turbulent logarithmic region. J Fluid Mech 2006; 561: 329-358.

Dukowicz, J, Dvinsky, A. Approximate factorization as a high order splitting for the implicit incompressible flow equations. J Comp Phys 1992; 102: 336-47.

Germano, M, Piomelli, U, Moin, P, Cabot, WH. A dynamic subgrid-scale eddy viscosity model. Phys. Fluids 1991; A3: 1760-5.

Ghosal, S. An analysis of numerical errors in large-eddy simulation of turbulence. J Comp Phys 1996; 125: 187-206. 
Hunt, JCR, Morrison, J. Eddy structures in turbulent boundary layers. Eur J Mech B Fluids 2000; 19: 673-94.

Jiménez, J. The largest structures in turbulent wall flows. Center for Turbulence Research Annual Research Briefs, Stanford University 1998; 137-54.

Kim, J, Moin, P, Moser, R. Turbulence statistics in fully developed channel flow at low Reynolds number. J Fluid Mech 1987; 177: 133-66.

Kim, KC, Adrian, RJ. Very large-scale motion in the outer layer. Phys Fluids $1999 ; 11,417-22$.

Komminaho, J, Lundbladh, A, Johansson, AV. Very large structures in plane turbulent Couette flow. J Fluid Mech 1996; 320: 259-85.

Lilly, DK. A proposed modification of the Germano subgrid-scale closure method. Phys Fluids 1992; A4: 633-5.

Meinhart, CD, Adrian, RJ. On the existence of uniform momentum zones in a turbulent boundary layer. Phys Fluids 1995; 7: 694-96.

Morinishi, Y, Lund, TS, Vasilyev, OV, Moin, P. Fully conservative higher order finite difference schemes for incompressible flow. J Comp Phys 1998; 143:

90-124.

Tsubokura, M. Proper representation of the subgrid-scale eddy viscosity for the dynamic procedure in large eddy simulation using finite difference method. Phys Fluids 2001; 13: 500-4.

Tsubokura, M. Subgrid scale modeling of turbulence for the dynamic procedure using finite difference method and its assessment on the thermally stratified 
turbulent channel flow. J of Applied Mechanics, Transactions of the ASME 2006;

73: $382-390$.

Tsubokura, M, Kobayashi, T, Taniguchi, N. Development of the isotropic eddy viscosity type SGS models for the dynamic procedure using finite difference method and its assessment on a plane turbulent channel flow. JSME Int J Ser B 2001; 44: 487-96.

Wei, T, Willmarth, WW. Reynolds-number effects on the structure of a turbulent channel flow. J Fluid Mech 1989; 204: 57-95.

Yoshizawa, A, Tsubokura, M, Kobayashi, T, Taniguchi, N. Modeling of the dynamic subgrid-scale viscosity in large eddy simulation. Phys Fluids 1996; 8, 2254-6. 


\section{FIGURE CAPTIONS}

Figure 1. Turbulence statistics at $R e_{\tau}=550$ (DNS) and 590 (LES): symbol, DNS; line, LES; (a) mean velocity; and (b) RMS of velocity fluctuations.

Figure 2. 1-D premultiplied power spectra of the streamwise velocity component at $y^{+} \sim 15$ : (a) streamwise; and (b) spanwise.

Figure 3. 1-D premultiplied power spectra of the streamwise velocity component at $y / \delta \sim 0.5$ : (a) streamwise; and (b) spanwise.

Figure 4. GS turbulence statistics of the open channel flows: (a) mean streamwise velocity; and (b) RMS velocity profiles in the inner scaling.

Figure 5. 1-D premultiplied power spectra of the streamwise velocity of the open channel flow at $y^{+} \sim 20$ against (a) streamwise and (b) spanwise wavelengths in the inner scaling.

Figure 6. 1-D premultiplied power spectra of the streamwise velocity of the open channel flows at $y / \delta \sim 0.5$ against (a) streamwise and (b) spanwise wavelengths in the outer scaling.

Figure 7. Instantaneous velocity-fluctuation fields of (a) streamwise, (b) normalwall, and (c) spanwise components of the open channel flows on an $x-z$ plane at $y / \delta \sim 0.5$ at $\operatorname{Re}_{\tau}=1180$. 
Figure 8. Instantaneous streamwise velocity fluctuations of the open channel flows on a $y-z$ plane at $R e_{\tau}=1180$ (positions on an $x-z$ plane are indicated in Fig. 7a).

Figure 9. Instantaneous streamwise velocity fluctuations of the open channel flows on an $x-z$ plane at $y^{+} \sim 20$ at $R e_{\tau}$ of (a) 395, (b) 590, and (c) 1180. The dotted oval in (c) indicates an example of the large darker spots that correlate closely with the large-scale structures observed in the outer layer (see Fig. 10c for the corresponding large scale).

Figure 10. Instantaneous streamwise velocity fluctuations of the open channel flows on an $x-z$ plane at $y / \delta \sim 0.5$ at $R e_{\tau}$ of (a) 395, (b) 590, and (c) 1180 .

Figure 11. Streamwise peak wavelength of the 1-D premultiplied power spectra of the open channel flows against distance from the wall: (a) inner scaling; and (b) outer scaling.

Figure 12. Spanwise peak wavelength of the 1-D premultiplied power spectra of the open channel flows against distance from the wall: (a) inner scaling; and (b) outer scaling. 


\section{Table 1}

Numerical conditions for the open channels

\begin{tabular}{|c|c|c|c|c|c|}
\hline \multirow[t]{2}{*}{$R e_{\tau}$} & \multirow{2}{*}{$\begin{array}{l}\text { Domain size } \\
L_{x} \times L_{y} \times L z\end{array}$} & \multirow{2}{*}{$\begin{array}{l}\text { Grid number } \\
N_{x} \times N_{y} \times N_{z}\end{array}$} & \multicolumn{3}{|c|}{ Grid spacing } \\
\hline & & & $h_{x}^{+}$ & $h_{y}^{+}$ & $h_{z}^{+}$ \\
\hline 395 & \multirow{3}{*}{$12 \pi \delta \times \delta \times 2.25 \pi \delta$} & $576 \times 32 \times 144$ & 25.9 & $0.9 \sim 29.6$ & 19.4 \\
\hline 590 & & $768 \times 48 \times 216$ & 29.0 & $0.8 \sim 29.6$ & 19.3 \\
\hline 1180 & & $1536 \times 64 \times 432$ & 29.0 & $1.1 \sim 46.2$ & 19.3 \\
\hline
\end{tabular}


Table 2

Numerical conditions for the solid plane channels in the grid resolution test

\begin{tabular}{lcccccc}
\hline \hline & \multirow{2}{*}{$e_{\tau}$} & Domain size & Grid number & \multicolumn{3}{c}{ Grid spacing } \\
& $=u_{\tau} \delta / \nu$ & $L_{x} \times L_{y} \times L_{z}$ & $N_{x} \times N_{y} \times N_{z}$ & $h_{x}^{+}$ & $h_{y}^{+}$ & $h_{z}^{+}$ \\
\hline (I) & 590 & $8 \pi \delta \times 2 \delta \times 4 \pi \delta$ & $192 \times 65 \times 192$ & 77.2 & $1.6 \sim 44.9$ & 38.6 \\
(II) & 590 & $8 \pi \delta \times 2 \delta \times 4 \pi \delta$ & $256 \times 65 \times 256$ & 57.9 & $1.6 \sim 44.9$ & 29.0 \\
(III) & 590 & $8 \pi \delta \times 2 \delta \times 4 \pi \delta$ & $384 \times 65 \times 384$ & 38.6 & $1.6 \sim 44.9$ & 19.3 \\
(IV) & 590 & $8 \pi \delta \times 2 \delta \times 4 \pi \delta$ & $512 \times 65 \times 512$ & 29.0 & $1.6 \sim 44.9$ & 14.5 \\
(V) & 590 & $8 \pi \delta \times 2 \delta \times 4 \pi \delta$ & $512 \times 65 \times 384$ & 29.0 & $1.6 \sim 44.9$ & 19.3 \\
DNS & 550 & $8 \pi \delta \times 2 \delta \times 4 \pi \delta$ & $1536 \times 257 \times 1536$ & 8.9 & $0.04 \sim 6.7$ & 4.5 \\
\hline \hline
\end{tabular}


Table 3

Reynolds numbers for the open channel flows.

\begin{tabular}{c|ccc}
\hline \hline$R e_{\tau}=u_{\tau} \delta / \nu$ & 395 & 590 & 1180 \\
\hline$R e_{C}=U_{C} \delta / \nu$ & $8.1 \times 10^{3}$ & $1.3 \times 10^{4}$ & $2.7 \times 10^{4}$ \\
\hline \hline
\end{tabular}



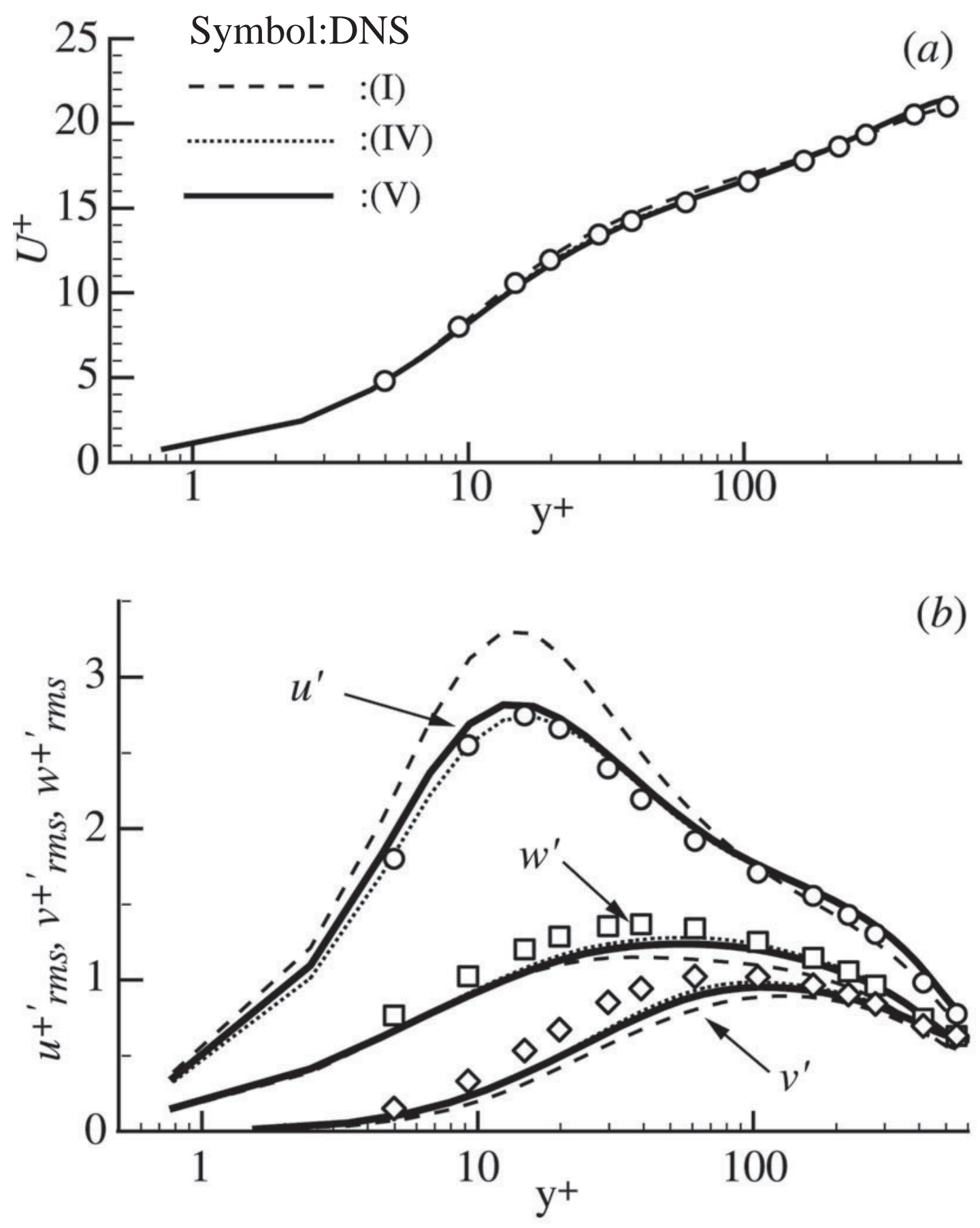

Fig. 1 Tsubokura 

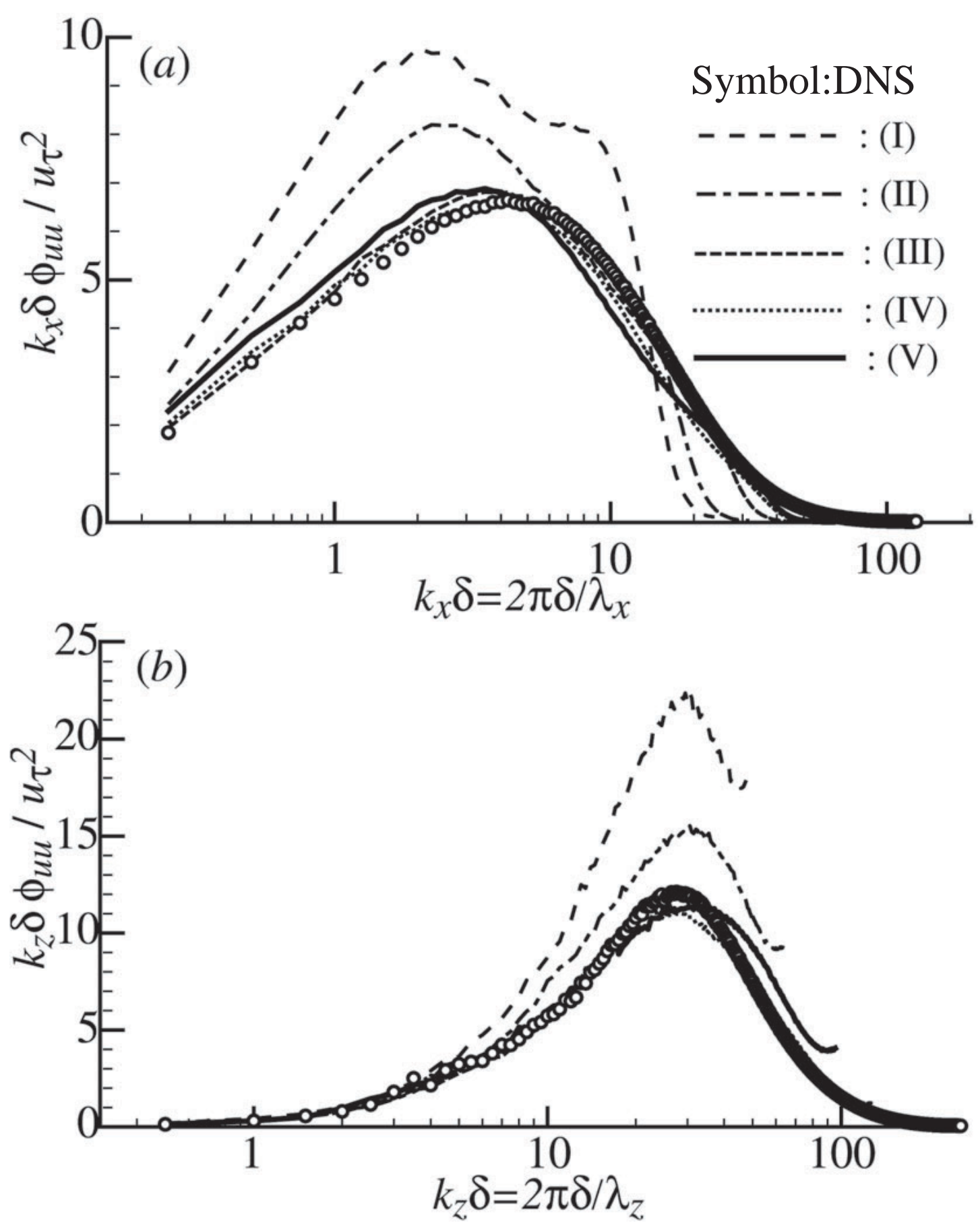

Fig. 2 Tsubokura 

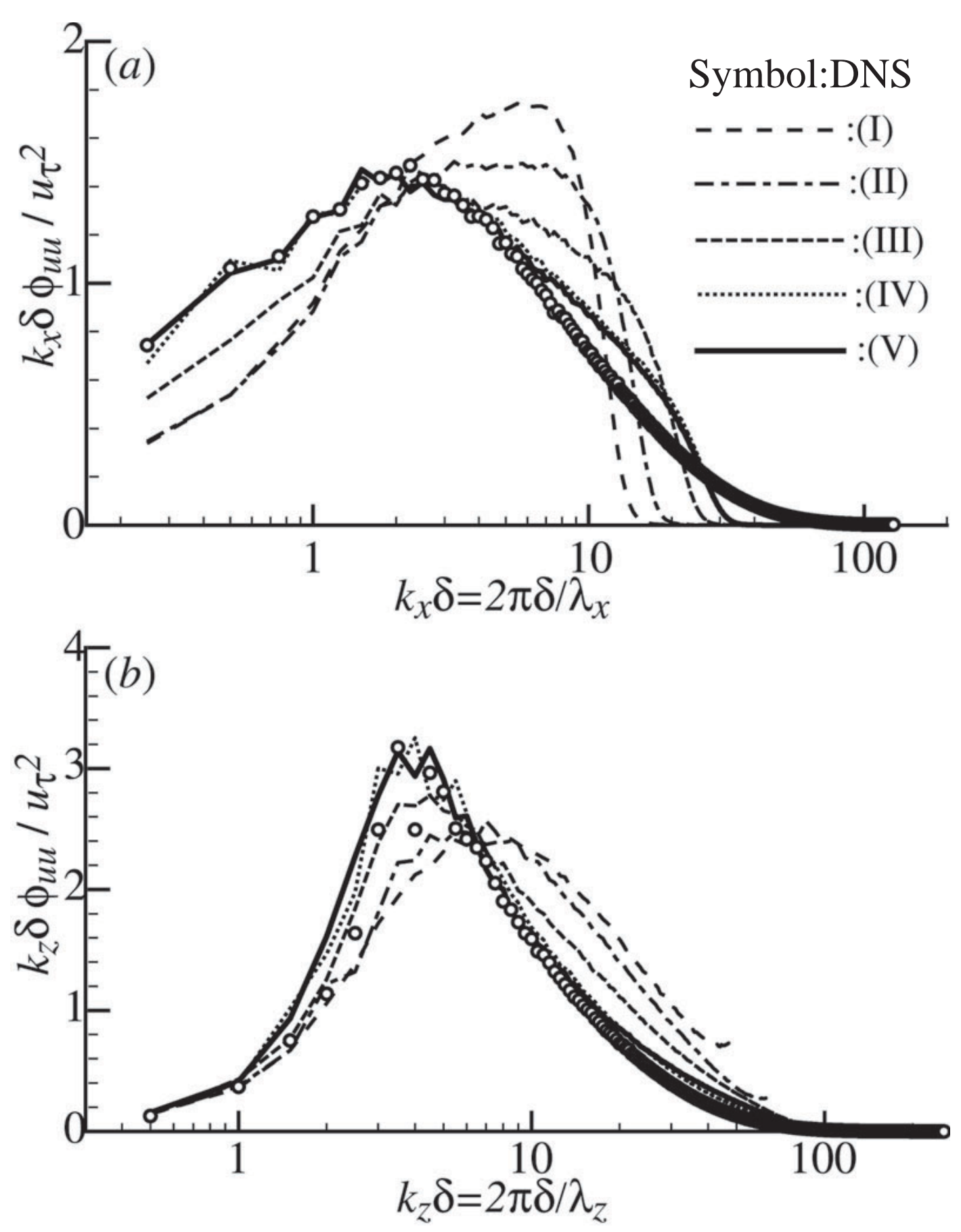

Fig. 3 Tsubokura 

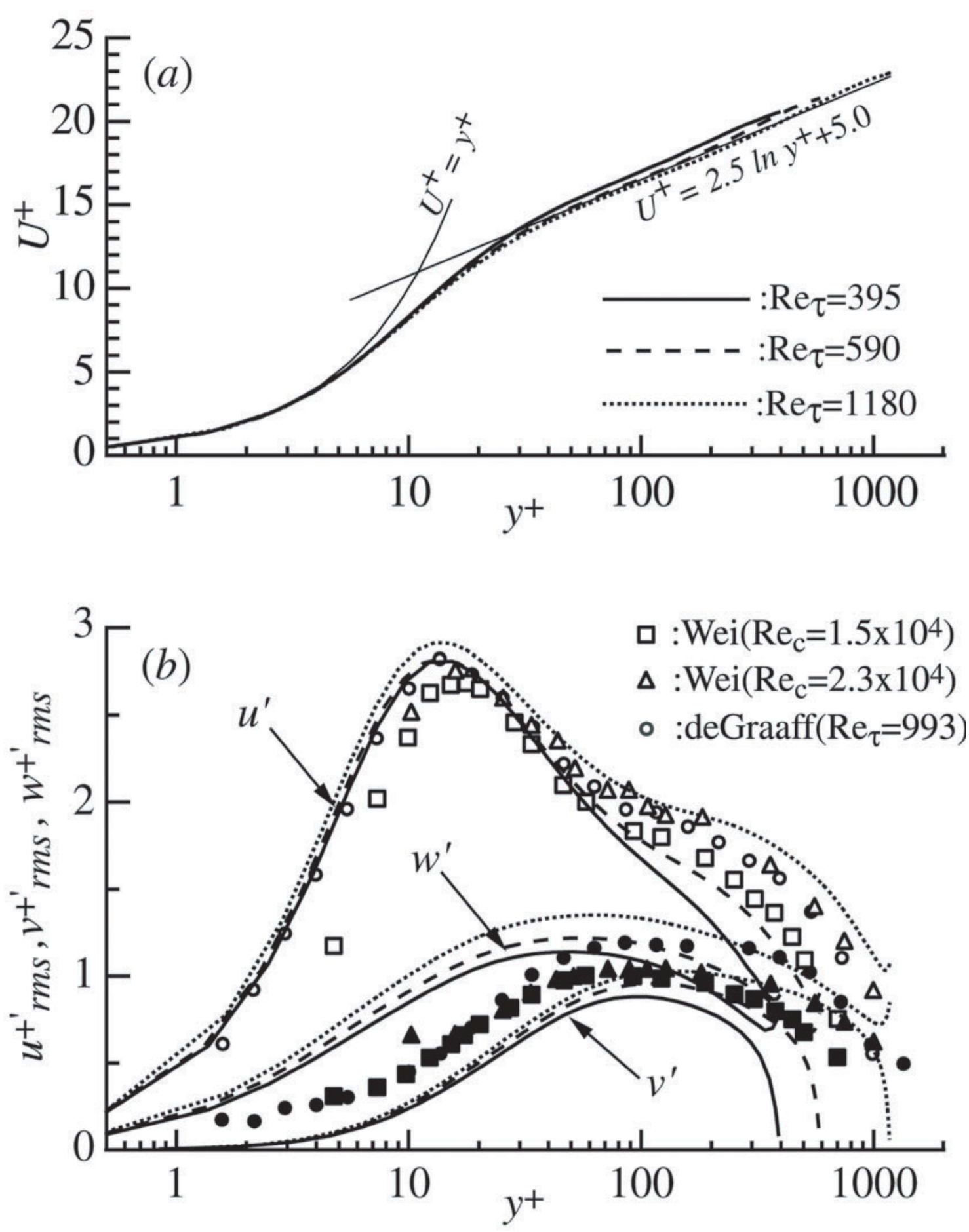

Fig. 4 Tsubokura 

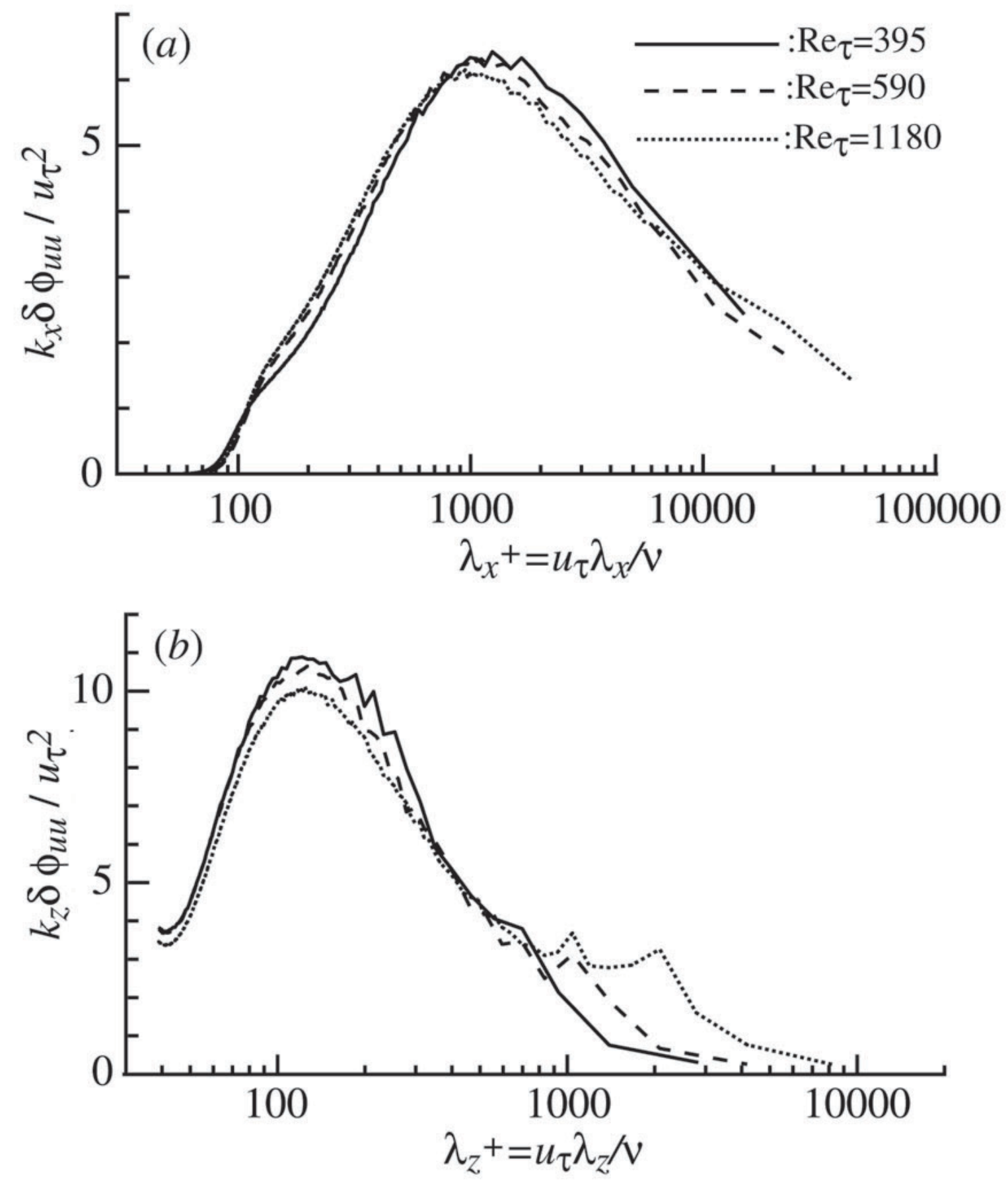

Fig. 5 Tsubokura 

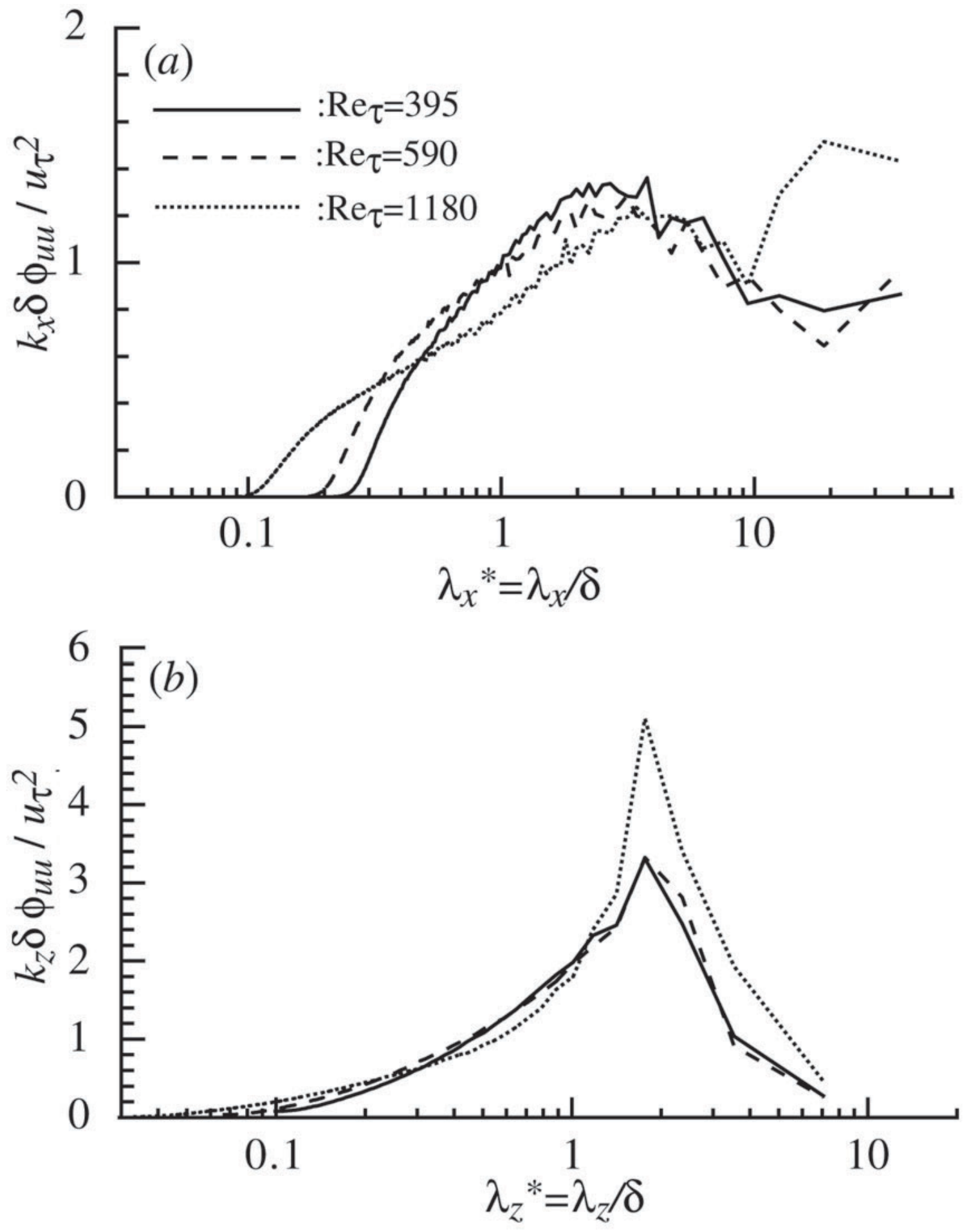

Fig. 6 Tsubokura 


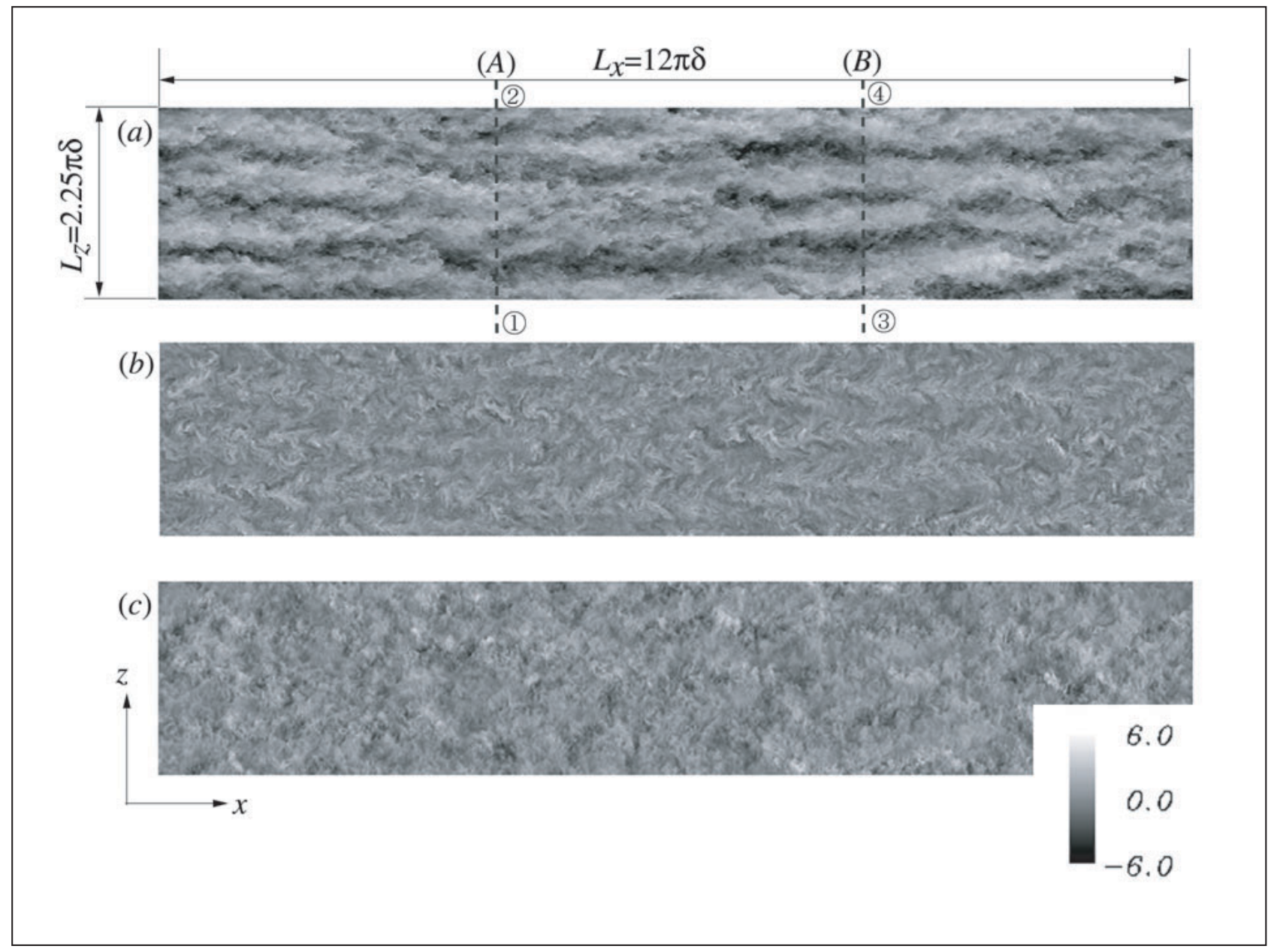

Fig. 7 Tsubokura 


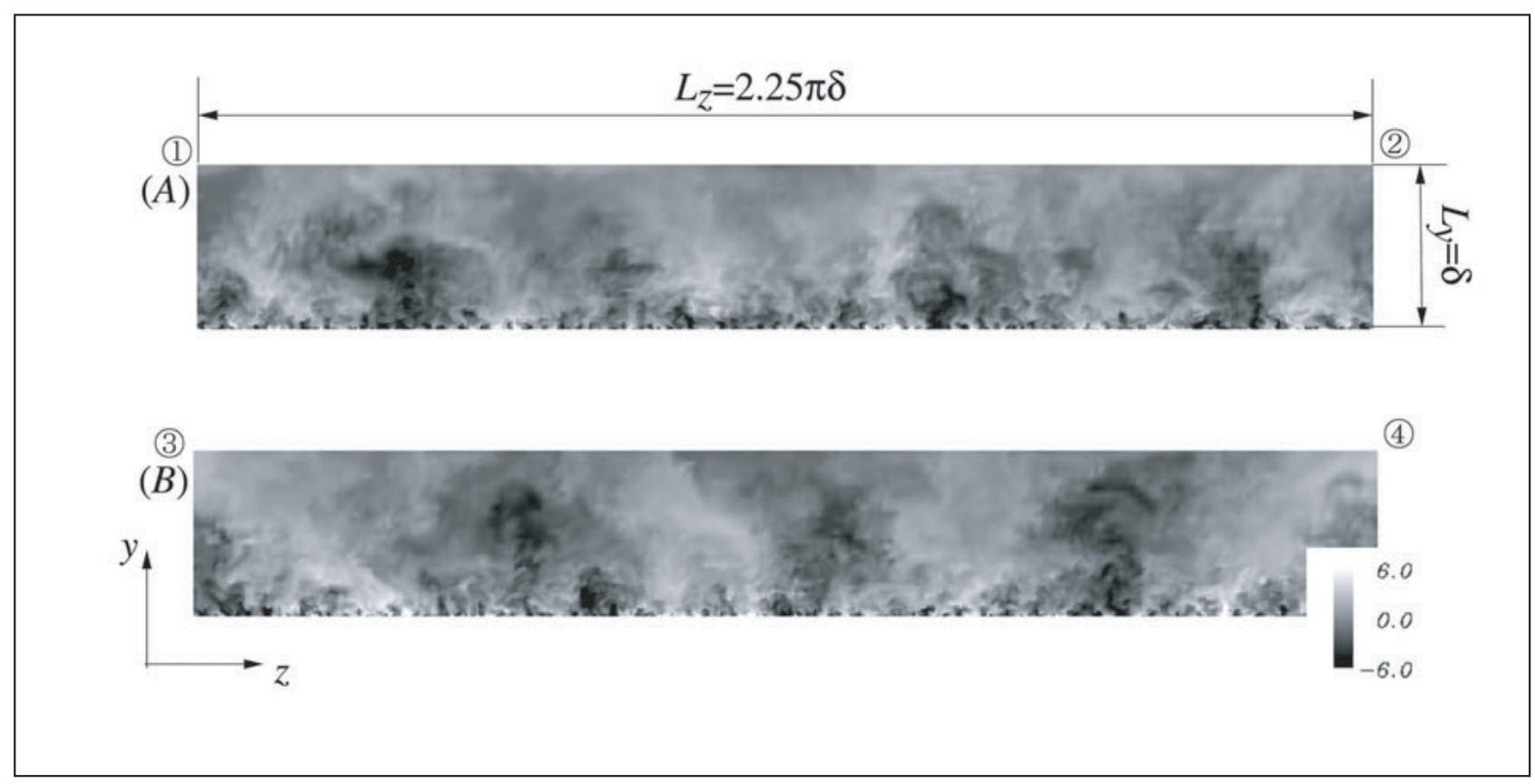

Fig. 8 Tsubokura 


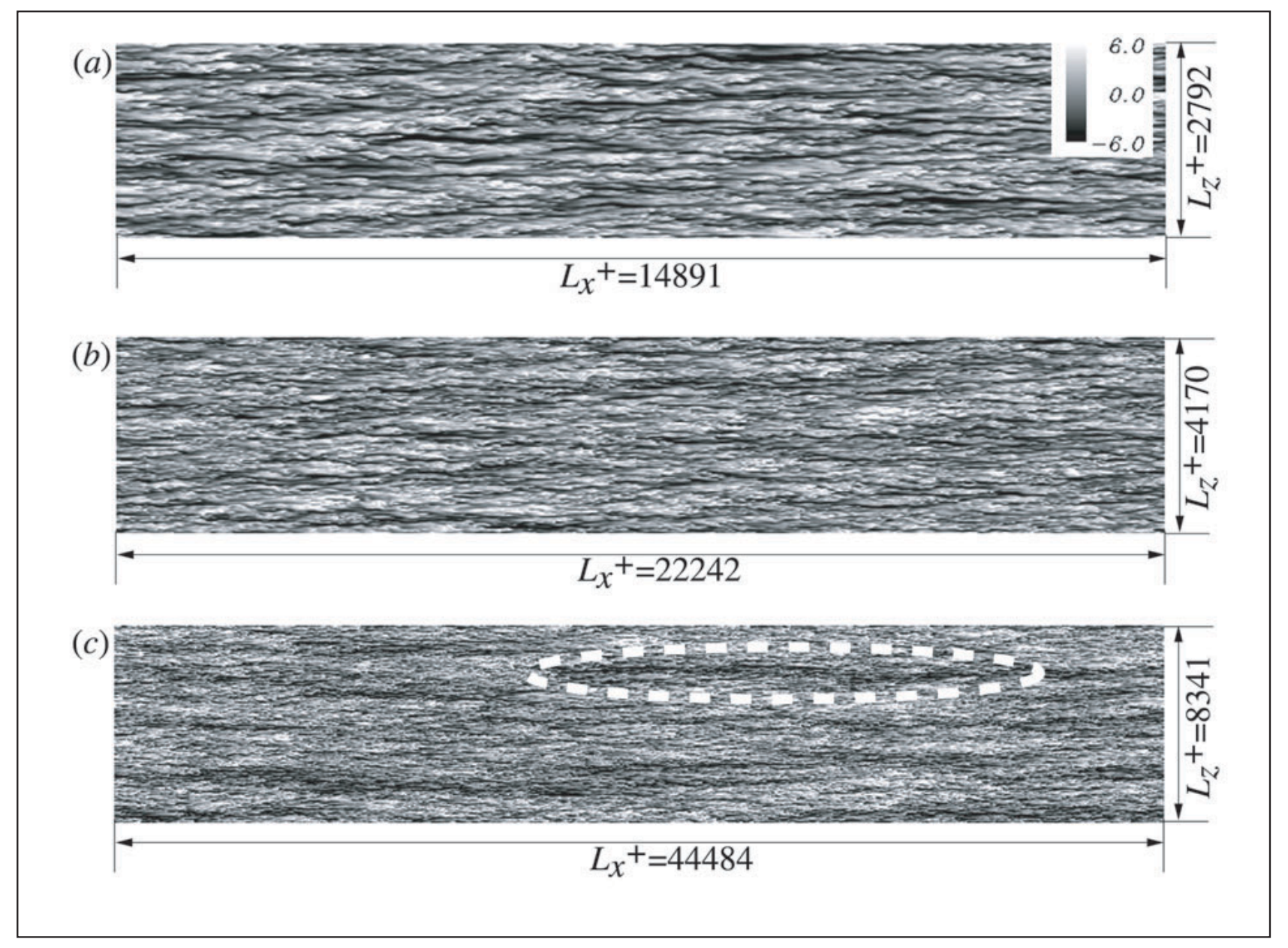

\section{Fig. 9 Tsubokura}




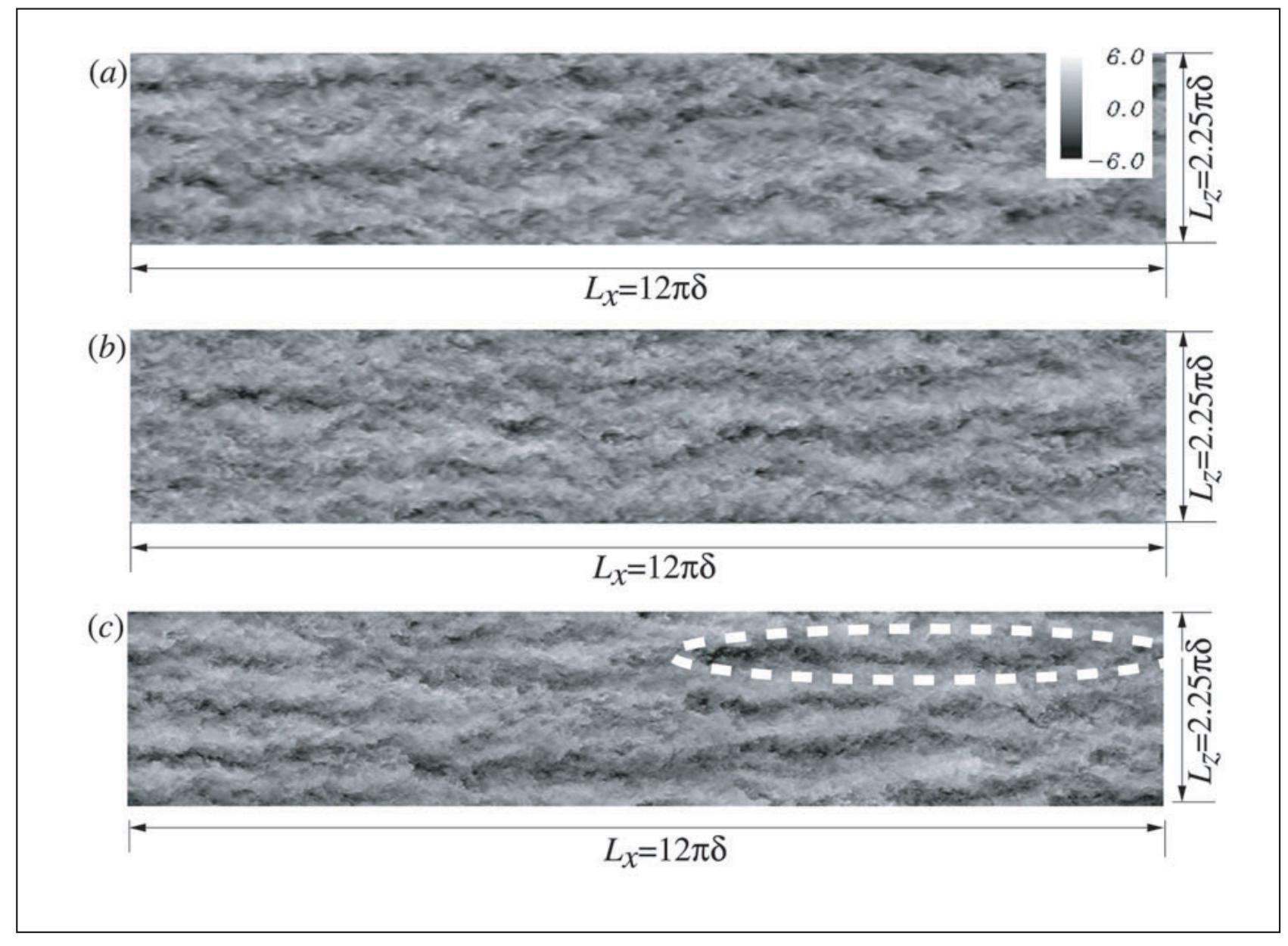

Fig. 10 Tsubokura 


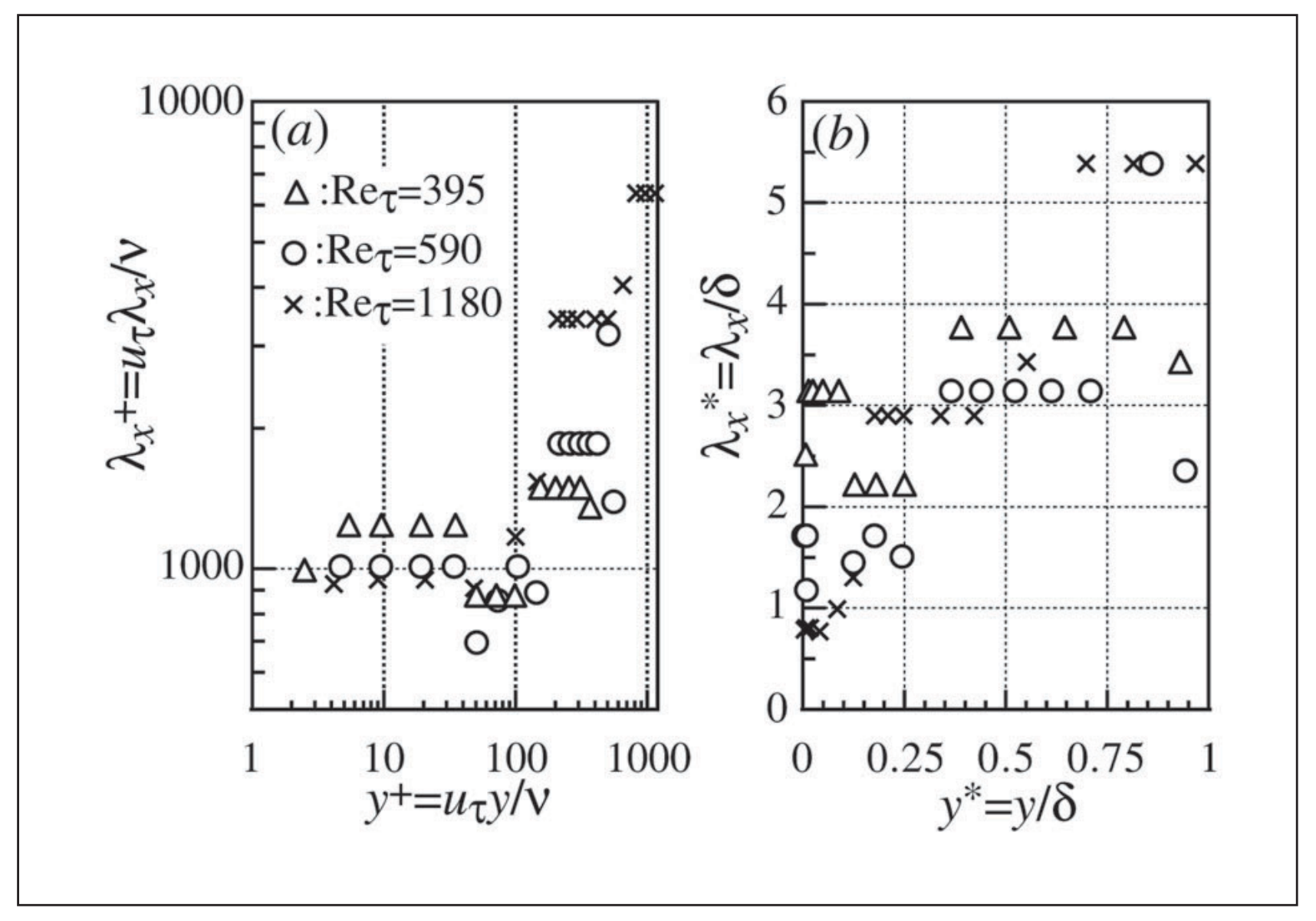

\section{Fig. 11 Tsubokura}




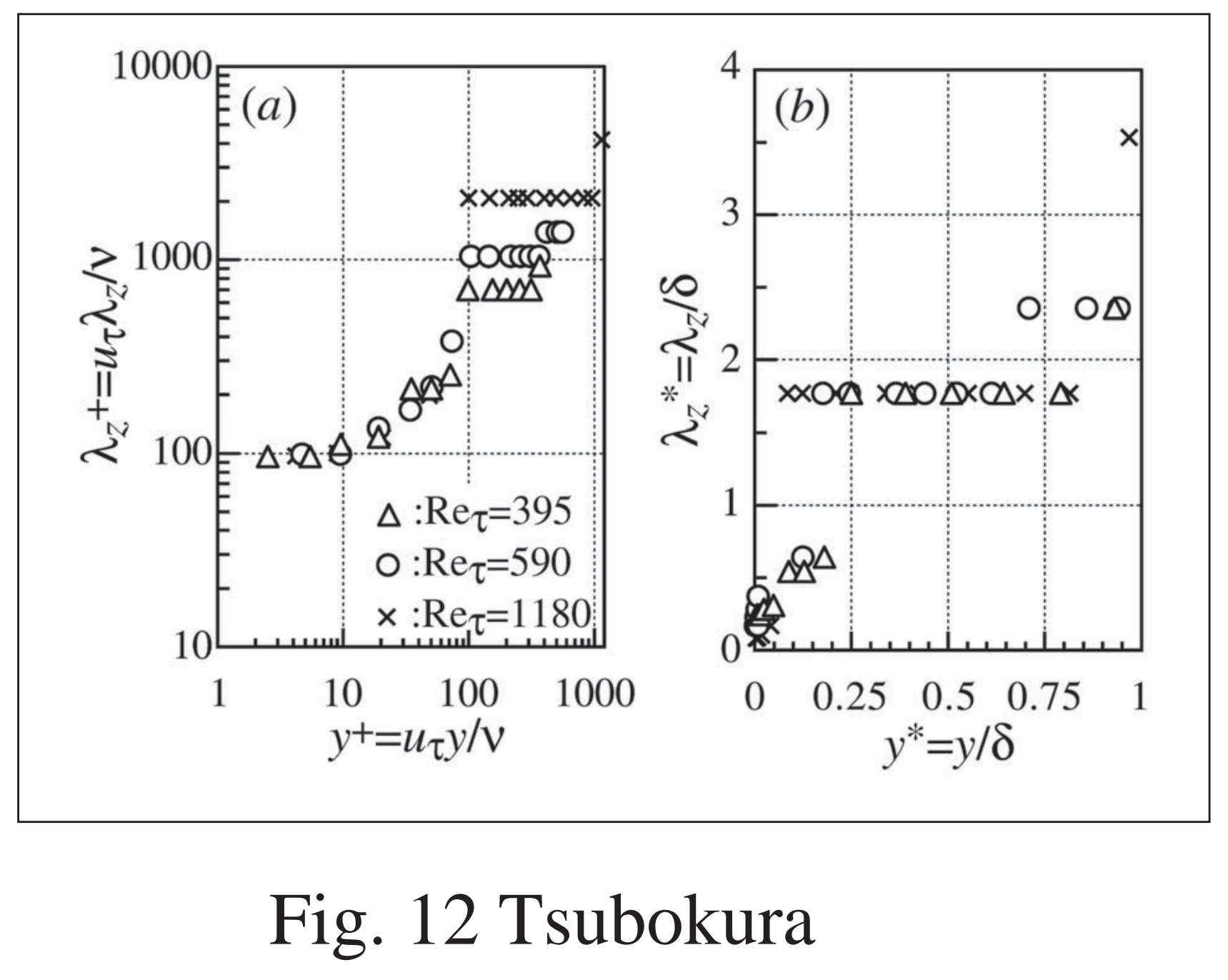

